\title{
Reuse-distance-based Miss-rate Prediction on a Per Instruction Basis
}

\author{
Changpeng Fang \\ cfang@mtu.edu
}

\author{
Steve Carr \\ carr@mtu.edu \\ Department of Computer Science \\ Michigan Technological University \\ Houghton MI 49931-1295 USA
}

Zhenlin Wang
zlwang@mtu.edu

\section{Keywords}

Data locality, reuse distance, profiling

\section{INTRODUCTION}

With the widening gap between processor and memory speeds, program performance has become increasingly dependent upon the effective use of a machine's memory hierarchy. To improve locality in programs, compilers have traditionally used either static analysis of regular array references $[8,13]$ or profiling [5] to determine the locality of memory operations. Static analysis has limited applicability when index arrays are used in addressing and when determining locality across multiple loop nests. Profiling can measure locality for a larger set of references, but the results may be specific to the profiled data set. This may lead to inaccurate analysis when the input set is changed.

Recently, Ding, et al. [2, 14], have proposed techniques to predict the reuse distance of memory references across all program inputs using a few profiling runs. They use curve fitting to predict reuse distance (the number of distinct memory locations accessed between two references to the same memory location) as a function of a program's data size. By quantifying reuse as a function of data size, the information obtained via a few profiled runs allow the prediction of reuse to be quite accurate over varied data sizes. Ding, et al., have used reuse-distance predictions to accurately predict whole program miss rates [14].

In order to use reuse distance in program optimization, it is beneficial to associate reuse distance prediction with the actual instructions that access those locations. By predicting the reuse distance for individual instructions, we can identify those instructions which may be most amenable to cache optimization. In this paper, we extend the work of Ding, et al., to apply reuse-distance prediction on a per instruction basis. We examine the reuse distance of the data referenced by each memory operation and use that information to determine the reuse distance of the operation as a function of the data size. We show experimentally that the reuse distance of static instructions is highly predictable. Furthermore, both the L1 and L2 miss rates for instructions can be predicted accurately using reuse-distance-based analysis.

Reuse-distance analysis opens up new possibilities for compiler and architectural optimization of memory operations. The dynamic analysis information provides the compiler with additional information that is impossible to compute at com- 
pile time, allowing the compiler to have more knowledge concerning the effects of optimization. Reuse-distance analysis may also be used in micro-architectural optimization via compiler hints to gain a more global view of the expected behavioral patterns of a program.

The most obvious application of reuse distance is to prefetching those memory operations that cause the most misses. Both hardware and software prefetching may issue many unnecessary prefetches. By predicting the miss rate of instructions, we can identify those instructions that are likely to provide the most benefit via prefetching and eliminate misses through dynamic or feedback-directed optimization. In addition, hardware could be constructed to use reusedistance information to schedule prefetches dynamically for important instructions.

We begin the rest of this paper with a review of work related to static and dynamic locality analysis. Next, we describe our analysis techniques and algorithms for measuring instruction-based reuse distance. Then, we present our experiments examining the predictability of instruction-based reuse distance and finish with our intentions for application of this analysis.

\section{RELATED WORK}

The previous work most pertinent to this paper consists of the two recent papers by Ding, et al. [2, 14]. They present a technique to predict reuse patterns of the whole program based on a couple of training runs on small inputs [2]. A follow-up work uses the predicted reuse distances to estimate the capacity miss rates of a fully associative cache [14]. We describe their techniques in detail in Section 3.1. In contrast, our work focuses on the prediction of reuse patterns and miss rates for each instruction. We expect to use this prediction to capture critical instructions, which generate dominant misses for a program.

Typically we can rely on cache simulation or an analytical model to analyze program cache behavior. Cache simulation can supply accurate miss rates and even performance impact for a cache configuration. The simulation itself is costly, making it less adaptive to system changes and impossible to apply during dynamic optimization on the fly. Various techniques have been discussed to make cache simulation more effective. Mattson, et al., present a stack algorithm to measure cache misses for different cache sizes in one run [6]. Sugumar and Abraham [12] use Belady's algorithm to characterize capacity and conflict misses. They present three techniques for fast simulation of optimal cache replacement. We find using the reuse-distance pattern to predict cache misses for other inputs yields better accuracy than applying cache simulation on a single test input.

A simple analytical model uses dependence and locality analysis to detect data reuse and estimate cache misses [4, 10]. McKinley, et al., design such a model to group reuses and estimate cache miss costs for loop transformations [8]. McKinley and Temam quantify loop nest locality related to self or group, spatial or temporal reuses [9]. Wolf and Lam use an approach based upon uniformly generated sets to analyze locality. Their technique produces similar results to that of McKinley, but does not require the storage of input dependences [13]. Beyls and D'Hollander detect reuse patterns through profiling and generate hints for the Itanium processor [5]. It's unclear whether their profiling and experiments are on the same input or not. However, our work can be directly applied to generate their hints.

Both the dependence based and uniformly generated set based locality analysis provide a high-level view of data reuse and data locality, but may generate poor accuracy, especially due to the lack of consideration of cache interference. For better cache interference analysis, Ghosh, et al. [3], suggest a set of miss equations for precisely analyzing cache misses for individual loop nests. Their framework enables compiler algorithms to find optimal solutions for transformations like blocking, loop fusion, and padding. Cache miss equations allow the compiler to reason about how different transformations work together. Chatterjee, et al. [1], set up a set of Presburger formulas to characterize and count cache misses. Chatterjee's model is powerful enough to handle imperfectly nested loops and various non-linear array layouts. Compared to cache simulation, these analytical models can capture high-level reuse patterns but may be hard to use for dynamic optimization due to lack of run-time knowledge such as loop bounds. Both the models of Ghosh and of Chatterjee sacrifice prediction efficiency for high accuracy.

\section{REUSE-DISTANCE ANALYSIS}

We begin this section with a description of reuse distance and whole-program locality analysis. Reuse distance provides the foundation for our analysis. We then describe per instruction reuse-distance and miss-rate analysis in detail.

\subsection{Reuse Distance}

The reuse distance of a reference is defined as the number of distinct memory references between itself and its reuse. In this paper, we ignore the impact of spatial locality by treating each cache line (block) as a unit, resulting in the measurement of reuse on a per cache line basis. Backward reuse distance is the gap between a particular memory reference and its previous access. Similarly, forward reuse distance quantifies the gap between a particular reference and the next access to the same location.

Previous work represents the whole program as a histogram describing reuse distance distribution, where each bar consists of the portion of memory references whose reuse distance falls into the same range [2]. Ding, et al., investigate dividing the consecutive ranges linearly, logarithmically, or simply by making the number of references in a range a fixed portion of total references. Our work uses logarithmic division where we put reuse distances between $2^{k}$ and $2^{k+1}$ into the same range. These bounds on the ranges are related to increments in cache size.

Ding, et al., define the data size of an input as the largest reuse distance. Given two histograms with different data sizes, they find the locality patterns of a third data size are predictable in a selected set of benchmarks. The pattern recognition step generates the histogram for the third input. Typically, one can use this method to predict locality patterns for a large data input of a program based on training runs of a pair of small inputs.

Let $d_{i}^{1}$ be the distance of the $i^{\text {th }}$ bin in the first histogram and $d_{i}^{2}$ be that in the second histogram. Assuming that $s_{1}$ and $s_{2}$ are the data sizes of two training inputs, we can fit the reuse distances through two coefficients, $c_{i}$ and $e_{i}$, and a function $f_{i}$ as follows.

$$
\begin{aligned}
& d_{i}^{1}=c_{i}+e_{i} * f_{i}\left(s_{1}\right) \\
& d_{i}^{2}=c_{i}+e_{i} * f_{i}\left(s_{2}\right)
\end{aligned}
$$


When the function $f_{i}$ is fixed, $c_{i}$ and $e_{i}$ can be calculated and the equation can be applied to another data size to predict reuse distance distribution. Ding, et al., try several types of fitting functions, such as linear or square root, and choose the best fit.

\subsection{Reuse Distance Prediction}

Although we know that the reuse distance distribution of the whole program is predictable, it is unclear whether the reuse distances of an instruction show the same predictability. Moreover, the reuse pattern for each instruction can serve as better information for both static and dynamic optimizations. In this section, we discuss our methods to predict instruction-based reuse patterns.

To apply per instruction reuse distance and miss rate prediction on the fly, it is critical to represent the locality patterns of training runs as simply as possible without sacrificing much prediction accuracy. For the training runs, we collect the reuse distances of each instruction and store the number of instances for each of the power of 2 intervals. We also record the minimum, maximum, and mean distance within each interval. An interval is active if there exists an occurrence of reuse in the interval. Our results in Section 4 show that most instructions contain just one or two active intervals. We note that at most 6 words of information are needed for most instructions in order to track their reuse intervals.

The ad hoc use of power of 2 as bounds for intervals may split the locality pattern of certain reuses across the interval bounds. We solve this problem through an additional scan of original intervals and merge any pair of adjacent intervals $i$ and $i+1$ if

$$
\min _{i+1}-\max _{i} \leq \max _{i}-\min _{i} .
$$

This inequality is true if the difference between the maximum distance in interval $i+1$ and the minimum distance in interval $i$ is no greater than the length of interval $i$. The merging process continues by testing whether the newly created interval should be merged with the next interval. We observe that this additional pass reflects the locality patterns of each instruction and notably improves prediction accuracy.

Following the prediction model discussed in Section 3.1, the reuse distance patterns of each instruction for a third input can be predicted through two training runs. For each instruction, we predict its $i^{t h}$ interval by fitting the $i^{t h}$ interval in each of the training runs. The fitting function is then used to solve the minimum, maximum, and mean distances of the current interval. Note that this prediction is simple and fast, making it a good candidate for inclusion in adaptive compilation.

For reuse distance prediction, we compute both the prediction coverage and the prediction accuracy. Prediction coverage indicates the percentage of instructions whose reuse distance distribution can be predicted. An instruction's reuse distance distribution can be predicted if and only if it occurs in both of the training runs and all of its reuse distance patterns are regular. A pattern is said to be regular if the pattern occurs in both training runs and its reuse distance does not decrease in the larger input size. Irregular patterns rarely occur in our test programs. Prediction accuracy indicates the percentage of covered instructions whose reuse distance distribution is correctly predicted by our model.

An instruction's reuse distance distribution is said to be correctly predicted if and only if all of its patterns are correctly predicted. In the experiments, we cross-validate this prediction by comparing the predicted locality intervals with the collected intervals through a real run. The prediction is said to be correct if both the predicted and observed patterns fall in the same $2^{k}$ interval or if the predicted intervals and the observed intervals overlap by at least $90 \%$. Given two patterns $A$ and $B$ such that B.min $<A \cdot \max \leq B \cdot \max$, we say that $A$ and $B$ overlap by at least $90 \%$ if

$$
\frac{A \cdot \max -\max (A \cdot \min , B \cdot \min )}{\max (B \cdot \max -B \cdot \min , A \cdot \max -A \cdot \min )} \geq 0.9 .
$$

\subsection{Miss Rate Prediction}

This section describes miss rate prediction for each instruction. We first describe our general methodology for miss rate prediction given a single cache. Then, we apply our model to multi-level, set-associative caches.

Given the size of a fully associative cache, we predict a cache miss for the next reference to a particular cache line if its forward reuse distance is greater than the cache size. This model catches the compulsory and capacity misses, but neglects conflict misses, which have previously been shown to be relatively less critical in some programs [7].

If the minimum distance of an interval is greater than the cache size, all accesses in the interval are considered as misses. When the cache size falls in the middle of an interval, we estimate the miss rates based on relative position of the size in the interval. In our experiments, we used an effective cache size equal to one-half of the actual cache size for all set-associative cache configurations, where the associativity is small $(\leq 4)$. For the fully associative configurations we used the actual size as the effective size. The prediction for L2 cache is identical to that for L1 cache with the predicted L1 cache hits filtered out.

In our analysis, an accurate miss-rate prediction for an instruction occurs if the predicted miss rate is within $5 \%$ of the actual miss rate gleaned through cache simulation using the same input. Although the predicted miss rate does not include conflict misses, the actual miss rate does. The results reported in the next section show that in spite of not incorporating conflict misses in the prediction, our prediction of miss rates is highly accurate.

\section{EXPERIMENT}

In this section, we report the results of our experiment evaluating the predictability of per instruction reuse distance. We begin with a discussion of our experimental methodology and then, we discuss the predictability of instruction reuse distance. Next, we report the predictability of per instruction cache miss rate for six different cache organizations. Finally, we show that our analysis can accurately predict the instructions that generate the most L2 misses.

\subsection{Methodology}

To compute reuse distance, we instrument the program binaries using Atom [11] to collect the data addresses for all memory instructions. The Atom scripts incorporate Ding and Zhong's reuse-distance collection tool [2, 14] into our analyzer to obtain reuse distances. During profiling, our 
analysis records the cache-line based forward reuse distance distribution for each individual memory instruction.

Our benchmark suite consists of 11 of the 14 programs from SPEC CFP2000. Table 1 lists the programs that we used. The remaining three benchmarks in SPEC CFP2000 are not included because we could not get them to compile correctly on our Alpha cluster. We used version 5.5 of the Compaq compilers using the -O3 optimization flag to compile the programs.

In the tables and figures reported throughout the rest of this section, we report both static and dynamic weightings of the results. The static weighting considers each instruction weighted identically, irrespective of the number of times it is executed. The dynamic weighting weights each static instruction by the number of times it executed. For instance, if a program contains two memory instructions, $A$ and $B$, and we correctly predict the result for instruction $A$ and incorrectly predict the result for instruction $B$, we have a $50 \%$ static prediction accuracy. If, however, instruction $A$ is executed 80 times and instruction $B$ is executed 20 times, we have an $80 \%$ dynamic prediction accuracy.

For miss-rate prediction measurements, we implemented a cache simulator and embedded it in our analysis routines to collect the number of L1 and L2 misses for each instruction. Table 2 shows the cache configurations that we used in our experiments. Each of the cache configurations uses 64-byte lines and an LRU replacement policy.

\begin{tabular}{|c|c|c|c|}
\hline config. no. & $\overline{\text { L1 }}$ & & L2 \\
\hline $\begin{array}{l}1 \\
2 \\
3\end{array}$ & $32 \mathrm{~K}, 2-$ way & $1 \mathrm{M}$ & $\begin{array}{c}\text { fully assoc } \\
4-\text { way } \\
2-\text { way }\end{array}$ \\
\hline $\begin{array}{l}4 \\
5 \\
6\end{array}$ & 16K, 2-way & $512 \mathrm{~K}$ & $\begin{array}{c}\text { fully assoc } \\
4-\text { way } \\
2-\text { way }\end{array}$ \\
\hline
\end{tabular}

Table 2: Cache configurations

To compare the effectiveness of our miss-rate prediction, we implemented three miss-rate prediction schemes. The first scheme, called predicted reuse distance (PRD), uses the reuse-distance predicted by our analysis of the training runs to predict the miss rate for each instruction. We use the test and train input sets for the training runs. The second scheme, called reference reuse distance (RRD), uses the actual reuse distance computed by running the program on the reference input data set to predict the miss rates. RRD represents an upper bound on the effectiveness of using reuse distance to predict cache-miss rates. The third scheme, called test cache simulation (TCS), uses the miss rates collected from running the test data input set on a cache simulator to predict the miss rate of the same program run on the reference input data set. Table 3 gives a summary of each technique for easy reference.

\subsection{Results}

In this paper, we only report statistics involving forward reuse distance prediction. The results for backward reuse distance prediction are similar. We begin this section with data related to reuse distance distribution prediction accuracy and coverage. Then, we show how well reuse distance prediction predicts both L1 and L2 miss rates for six different cache configurations. Throughout the section, we focus more on the dynamically weighted results than the statically weighted results since the dynamic results factor in execution frequency.

\subsubsection{Reuse-distance Prediction Accuracy and Cov- erage}

Table 1 lists the coverage and accuracy of reuse-distance prediction on a per instruction basis. The statically weighted coverage is $89.9 \%$ while the dynamically weighted coverage is $91.3 \%$, on average. For all programs except 188.ammp and 189.lucas the dynamic coverage is well over $90 \%$. 188.ammp exhibits a larger than normal percentage of irregular patterns. In 189 .lucas, approximately $31 \%$ of the memory operations do not appear in both training runs. If an instruction does not appear during execution for both the test and train data sets, we cannot predict its reuse distance.

Our model predicts reuse distance correctly for $97.2 \%$ of the covered instructions using a static weighting and $96.7 \%$ using a dynamic weighting, on average. Our analysis predicts the reuse distance accurately for over $95 \%$ of the covered instructions using dynamic weighting for all programs except 189.lucas and 177.mesa. In 189.lucas, the fact that all instructions do not appear in both the test and train runs throws off our computation of reuse distance. These extra instructions change the reuse distance because different memory locations are accessed. In 177.mesa, some instructions that exhibit a single pattern in the test and train runs, exhibit multiple patterns in the reference run.

In Table 1, the column labeled "Patterns" shows the major patterns identified in the programs by our analysis. ${ }^{1}$ It can be seen that, for all programs, most patterns are constant patterns. However, a significant number of other patterns exist in some programs. For example, in 171.swim $12.3 \%$ of the patterns exhibit a square root (sqrt) distribution pattern and in 189 .lucas $16.7 \%$ of the patterns exhibit a linear distribution. For 200.sixtrack, we do not report the patterns since all data sizes are identical. This makes it impossible to construct a fitting function.

In addition to measuring the prediction coverage and accuracy, we measured the number of locality intervals exhibited by each instruction. Table 4 below shows the percentage of instructions that exhibit 1, 2 or 3 or more intervals during execution.

\begin{tabular}{|l|c|c|c|c|c|c|}
\hline \multirow{2}{*}{ Benchmark } & \multicolumn{3}{|c|}{ Static Weighting } & \multicolumn{3}{|c|}{ Dynamic Weighting } \\
\cline { 2 - 7 } & 1 & 2 & $\geq 3$ & 1 & 2 & $\geq 3$ \\
\hline 188.ammp & 83.8 & 13.3 & 2.9 & 78.5 & 18.0 & 3.5 \\
\hline 173.applu & 73.8 & 23.3 & 2.9 & 62.7 & 32.5 & 4.8 \\
\hline 301.apsi & 87.2 & 10.0 & 2.8 & 60.3 & 31.3 & 8.4 \\
\hline 179.art & 92.8 & 6.4 & 0.8 & 91.1 & 8.8 & 0.1 \\
\hline 183.equake & 90.7 & 7.6 & 1.6 & 81.0 & 16.5 & 2.5 \\
\hline 189.lucas & 81.7 & 17.5 & 0.8 & 65.5 & 33.9 & 0.6 \\
\hline 177.mesa & 91.0 & 7.1 & 1.9 & 86.2 & 10.3 & 3.5 \\
\hline 172.mgrid & 70.4 & 17.1 & 12.5 & 60.4 & 25.0 & 14.6 \\
\hline 200.sixtrack & 84.2 & 23.1 & 2.7 & 64.8 & 22.1 & 13.1 \\
\hline 171.swim & 72.6 & 27.2 & 0.2 & 49.9 & 49.5 & 0.6 \\
\hline 168.wupwise & 91.5 & 6.7 & 1.8 & 66.2 & 22.1 & 11.7 \\
\hline average & 83.6 & 14.5 & 2.8 & 69.7 & 24.5 & 5.8 \\
\hline
\end{tabular}

\section{Table 4: Number of locality intervals}

For our benchmark suite, all but three programs have more than $90 \%$ of their instructions exhibiting only one or two reuse-distance patterns. Each of the other three programs

\footnotetext{
${ }^{1}$ Note that the rows labeled "1third" indicate $f_{i}(s)=s^{\frac{1}{3}}$.
} 


\begin{tabular}{|c|c|c|c|c|c|c|c|}
\hline \multirow[b]{2}{*}{ Benchmark } & \multirow[b]{2}{*}{ Patterns } & \multirow[b]{2}{*}{ Input Set } & \multirow[b]{2}{*}{$\begin{array}{l}\text { Data Size } \\
\text { (cache blocks) }\end{array}$} & \multicolumn{2}{|c|}{ Static Weighting } & \multicolumn{2}{|c|}{ Dynamic Weighting } \\
\hline & & & & $\begin{array}{c}\text { Coverage } \\
(\%)\end{array}$ & $\begin{array}{c}\text { Accuracy } \\
(\%)\end{array}$ & $\begin{array}{c}\text { Coverage } \\
(\%)\end{array}$ & $\begin{array}{c}\text { Accuracy } \\
(\%)\end{array}$ \\
\hline 188.ammp & $\begin{array}{l}\text { const }(87.1 \%) \\
\text { linear }(9.8 \%) \\
\text { others }(3.9 \%)\end{array}$ & $\begin{array}{l}\text { test*(1002 atoms }) \\
\text { train(30002 atoms }) \\
\text { ref(300002 atoms })\end{array}$ & $\begin{array}{l}7.2 \mathrm{~K} \\
147.1 \mathrm{~K} \\
366.7 \mathrm{~K}\end{array}$ & 89.9 & 97.0 & 84.1 & 97.0 \\
\hline 173.applu & $\begin{array}{l}\text { const }(87.3 \%) \\
1 \text { third }(6.8 \%) \\
\text { others }(5.9 \%)\end{array}$ & $\begin{array}{l}\operatorname{test}(12 \times 12 \times 12) \\
\operatorname{train}(24 \times 24 \times 24) \\
\operatorname{ref}(60 \times 60 \times 60)\end{array}$ & $\begin{array}{l}18.8 \mathrm{~K} \\
175.1 \mathrm{~K} \\
2.86 \mathrm{M}\end{array}$ & 87.7 & 97.8 & 95.6 & 98.2 \\
\hline 301.apsi & $\begin{array}{l}\text { const }(86.9 \%) \\
\text { linear }(8.7 \%) \\
\text { others }(4.4 \%)\end{array}$ & $\begin{array}{l}\operatorname{test}^{*}(128 \times 1 \times 32) \\
\operatorname{train}(128 \times 1 \times 64) \\
\operatorname{ref}^{*}(128 \times 1 \times 256)\end{array}$ & $\begin{array}{l}8.2 \mathrm{~K} \\
15.1 \mathrm{~K} \\
62.3 \mathrm{~K}\end{array}$ & 96.0 & 98.6 & 92.1 & 96.6 \\
\hline 179.art & $\begin{array}{l}\text { const }(92.4 \%) \\
\text { linear }(3.9 \%) \\
\text { others }(3.7 \%)\end{array}$ & $\begin{array}{l}\text { test }(1 \text { object }) \\
\operatorname{train} *(10 \text { objects }) \\
\text { ref* }(30 \text { objects })\end{array}$ & $\begin{array}{l}37.4 \mathrm{~K} \\
57.4 \mathrm{~K} \\
107.4 \mathrm{~K}\end{array}$ & 82.8 & 98.4 & 99.9 & 99.9 \\
\hline 183.equake & $\begin{array}{l}\text { const }(86.6 \%) \\
1 \text { third }(5.3 \%) \\
\text { others }(7.9 \%)\end{array}$ & $\begin{array}{l}\text { test*(512 nodes }) \\
\text { train( } 7294 \text { nodes }) \\
\text { ref(30169 nodes })\end{array}$ & $\begin{array}{l}11.4 \mathrm{~K} \\
158.8 \mathrm{~K} \\
782.6 \mathrm{~K}\end{array}$ & 96.5 & 99.2 & 97.4 & 99.2 \\
\hline 189.lucas & $\begin{array}{l}\text { const }(81.7 \%) \\
\text { linear }(16.7 \%) \\
\text { others }(1.6 \%)\end{array}$ & $\begin{array}{l}\text { test } \\
\text { train } \\
\text { ref }\end{array}$ & $\begin{array}{l}782 \\
4.7 \mathrm{~K} \\
2.3 \mathrm{M}\end{array}$ & 66.7 & 93.9 & 52.2 & 88.4 \\
\hline 177.mesa & $\begin{array}{l}\text { const }(95.2 \%) \\
\text { linear }(2.2 \%) \\
\text { others }(5.6 \%)\end{array}$ & $\begin{array}{l}\operatorname{test}^{*}(200 \times 200) \\
\operatorname{train} *(400 \times 400) \\
\operatorname{ref}(800 \times 800)\end{array}$ & $\begin{array}{l}5.2 \mathrm{~K} \\
14.9 \mathrm{~K} \\
44.3 \mathrm{~K}\end{array}$ & 94.8 & 96.5 & 95.2 & 91.3 \\
\hline 172.mgrid & $\begin{array}{l}\text { const }(82.8 \%) \\
1 \text { third }(7.8 \%) \\
\text { others }(9.6 \%)\end{array}$ & $\begin{array}{l}\operatorname{test}^{*}(32 \times 32 \times 32) \\
\operatorname{train}(64 \times 64 \times 64) \\
\operatorname{ref}(128 \times 128 \times 128)\end{array}$ & $\begin{array}{l}16.6 \mathrm{~K} \\
119.5 \mathrm{~K} \\
907.4 \mathrm{~K}\end{array}$ & 90.3 & 94.0 & 94.2 & 95.6 \\
\hline 200.sixtrack & & $\begin{array}{l}\text { test } \\
\text { train } \\
\text { ref }\end{array}$ & $\begin{array}{l}391.8 \mathrm{~K} \\
391.8 \mathrm{~K} \\
391.8 \mathrm{~K}\end{array}$ & 99.9 & 99.7 & 99.7 & 99.9 \\
\hline 171.swim & $\begin{array}{l}\text { const }(71.2 \%) \\
\text { sqrt }(12.3 \%) \\
\text { others }(16.5 \%)\end{array}$ & $\begin{array}{l}\text { test }^{*}(128 \times 128) \\
\operatorname{train}(512 \times 512) \\
\operatorname{ref}(1334 \times 1334)\end{array}$ & $\begin{array}{l}32.7 \mathrm{~K} \\
476.4 \mathrm{~K} \\
3.1 \mathrm{M}\end{array}$ & 86.3 & 95.1 & 93.7 & 99.8 \\
\hline 168.wupwise & $\begin{array}{l}\text { const }(96.0 \%) \\
\text { 1third }(2.7 \%) \\
\text { others }(1.3 \%)\end{array}$ & $\begin{array}{l}\operatorname{test}^{*}(16 \times 5 \times 5 \times 5) \\
\operatorname{train} *(16 \times 10 \times 10 \times 10) \\
\operatorname{ref}(16 \times 20 \times 20 \times 20)\end{array}$ & $\begin{array}{l}45.1 \mathrm{~K} \\
360.1 \mathrm{~K} \\
2.9 \mathrm{M}\end{array}$ & 98.2 & 99.5 & 99.9 & 98.0 \\
\hline average & & & & 89.9 & 97.2 & 91.3 & 96.7 \\
\hline
\end{tabular}

Table 1: Forward reuse distance distribution prediction coverage and accuracy for CFP2000 benchmarks

\begin{tabular}{|l|l|l|}
\hline Scheme & Name & Description \\
\hline PRD & predicted reuse distance & Use reuse distance predicted from data size \\
\hline RRD & reference reuse distance & Use actual reference reuse distance \\
\hline TCS & test cache simulation & Use miss rates from test run \\
\hline
\end{tabular}

Table 3: Miss-rate prediction schemes

have over $85 \%$ of their instructions having only one or two reuse-distance patterns. This information shows that most references have highly predictable reuse patterns.

\begin{tabular}{|l|c|c|c|c|c|}
\hline Benchmark & $<1 \mathrm{~K}$ & $1-8 \mathrm{~K}$ & $8-32 \mathrm{~K}$ & $32-64 \mathrm{~K}$ & $\geq 64 \mathrm{~K}$ \\
\hline 188.ammp & 95.5 & 8.3 & 10.8 & 6.4 & 5.3 \\
\hline 173.applu & 98.2 & 1.7 & 2.2 & 1.9 & 7.4 \\
\hline 301.apsi & 95.9 & 4.6 & 6.1 & 3.6 & 0.0 \\
\hline 179.art & 86.3 & 0.3 & 16.6 & 6.2 & 6.2 \\
\hline 183.equake & 95.8 & 2.5 & 3.5 & 2.9 & 9.6 \\
\hline 189.lucas & 93.6 & 2.3 & 2.4 & 2.1 & 19.2 \\
\hline 177.mesa & 98.6 & 0.7 & 0.1 & 5.0 & 0.0 \\
\hline 172.mgrid & 97.9 & 14.8 & 13.1 & 6.6 & 17.4 \\
\hline 200.sixtrack & 95.8 & 3.1 & 4.4 & 3.1 & 2.6 \\
\hline 171.swim & 92.2 & 7.2 & 6.4 & 8.3 & 30.0 \\
\hline 168.wupwise & 96.6 & 0.4 & 0.2 & 0.4 & 5.1 \\
\hline average & 95.1 & 4.2 & 6.0 & 4.2 & 9.3 \\
\hline
\end{tabular}

Table 5: Percentage instructions per interval
Table 5 reports the distribution of the static instructions within the $2^{k}$ intervals for the reference data set using 64 byte cache lines. The table gives the percentage of instructions that have a reuse distance pattern that falls into each of the intervals. Note that instructions can fall into more than one interval. As can be seen from Table 5, most reuse patterns have a small reuse distance. However, there are a number of reuse patterns with a long reuse distance. These longer reuse distance instructions represent opportunities for cache optimizations as the likelihood of cache misses is higher.

To evaluate the effect of merging two intervals as discussed in Section 3.2, we report how often instructions whose reuse pattern crosses the $2^{k}$ boundaries are merged into a single pattern. Table 6 shows that in most programs less than $10 \%$ of the patterns are merged. Although not dominant, the merging still has a significant effect on our prediction mechanism. 


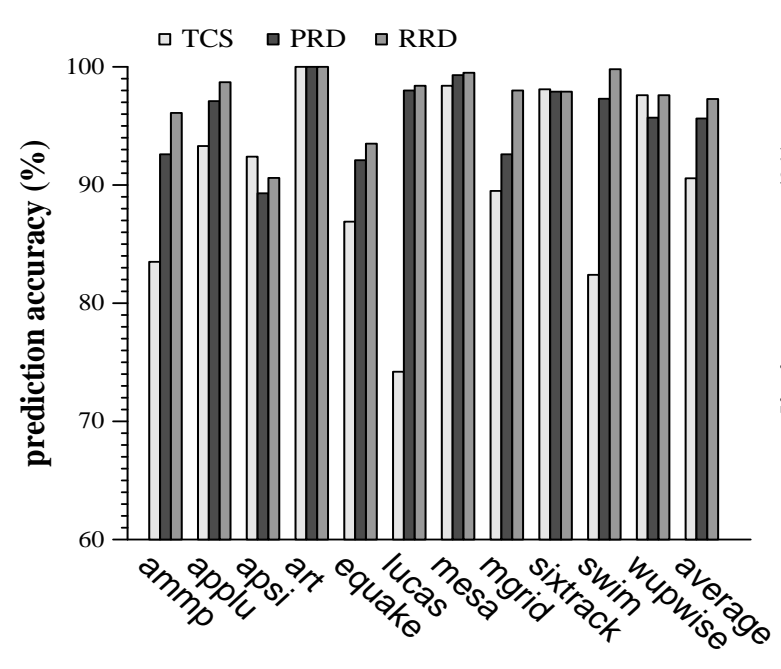

(a) L1 cache

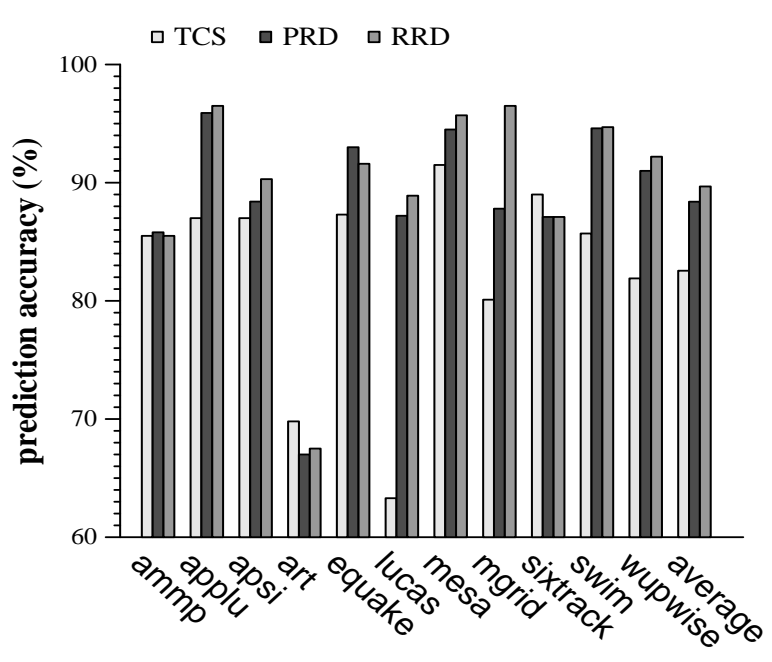

(c) 4-way set associative L2 cache

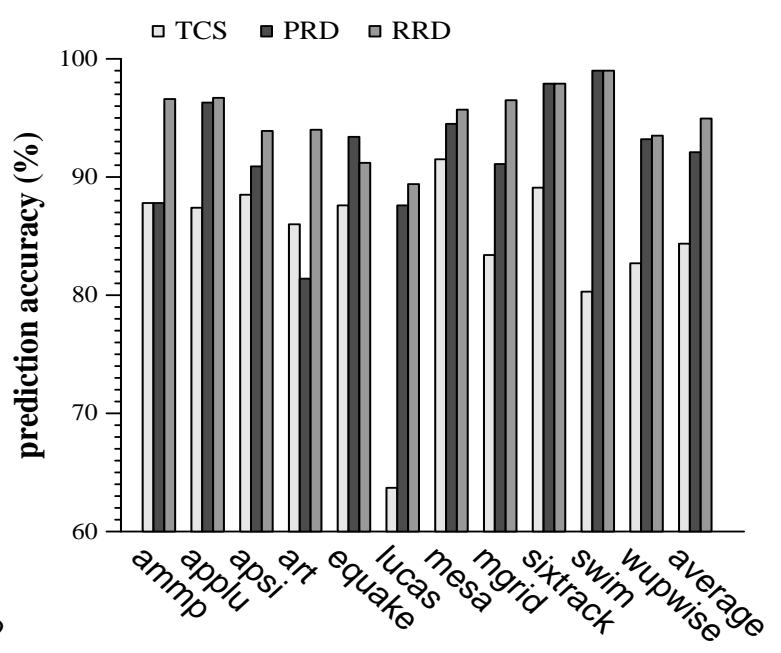

(b) Fully associative L2 cache

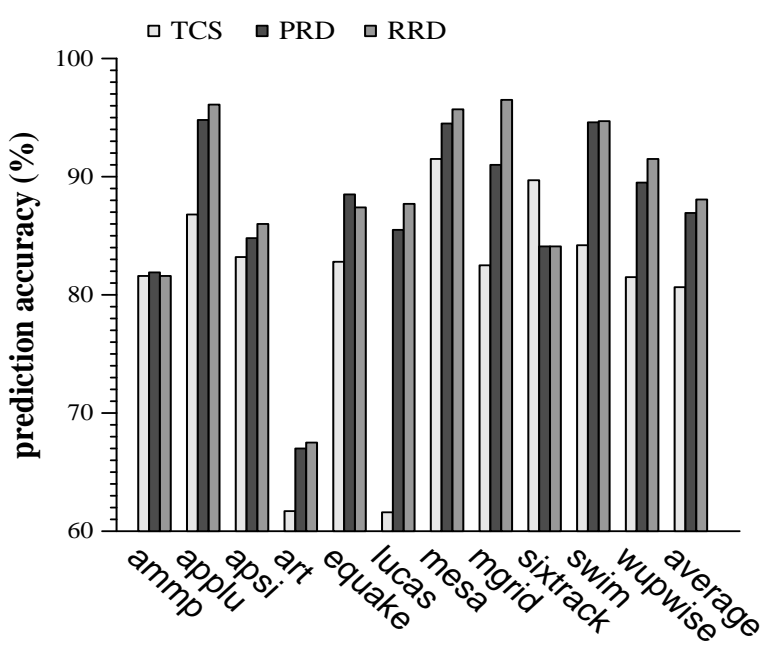

(d) 2-way set associative L2 cache

Figure 1: Dynamic miss-rate prediction accuracy

\begin{tabular}{|c|c|c|c|c|c|c|c|c|c|c|c|c|}
\hline \multirow{3}{*}{$\begin{array}{c}\text { Cache } \\
\text { Config. No. }\end{array}$} & \multicolumn{6}{|c|}{ L1 } & \multicolumn{6}{|c|}{$\overline{\mathbf{L 2}}$} \\
\hline & \multicolumn{3}{|c|}{ Dynamic Weighting } & \multicolumn{3}{|c|}{ Static Weighting } & \multicolumn{3}{|c|}{ Dynamic Weighting } & \multicolumn{3}{|c|}{ Static Weighting } \\
\hline & TCS & PRD & RRD & TCS & PRD & RRD & TCS & PRD & RRD & TCS & PRD & RRD \\
\hline 1 & \multirow{3}{*}{90.6} & \multirow{3}{*}{95.6} & \multirow{3}{*}{97.3} & \multirow{3}{*}{94.0} & \multirow{3}{*}{96.2} & \multirow{3}{*}{97.7} & 84.0 & 92.5 & 94.8 & 91.2 & 96.2 & 97.7 \\
\hline 2 & & & & & & & 82.6 & 88.4 & 89.7 & 90.7 & 94.9 & 96.3 \\
\hline 3 & & & & & & & 80.6 & 87.4 & 88.7 & 90.4 & 94.5 & 95.9 \\
\hline 4 & \multirow{3}{*}{90.3} & \multirow{3}{*}{93.1} & \multirow{3}{*}{94.5} & \multirow{3}{*}{93.8} & \multirow{3}{*}{94.8} & \multirow{3}{*}{96.2} & 85.9 & 92.4 & 93.8 & 92.0 & 96.0 & 97.4 \\
\hline 5 & & & & & & & 84.0 & 89.9 & 90.3 & 90.4 & 94.9 & 96.0 \\
\hline 6 & & & & & & & 82.1 & 87.7 & 88.0 & 90.9 & 94.3 & 95.3 \\
\hline
\end{tabular}

Table 7: Average miss-rate prediction accuracy 


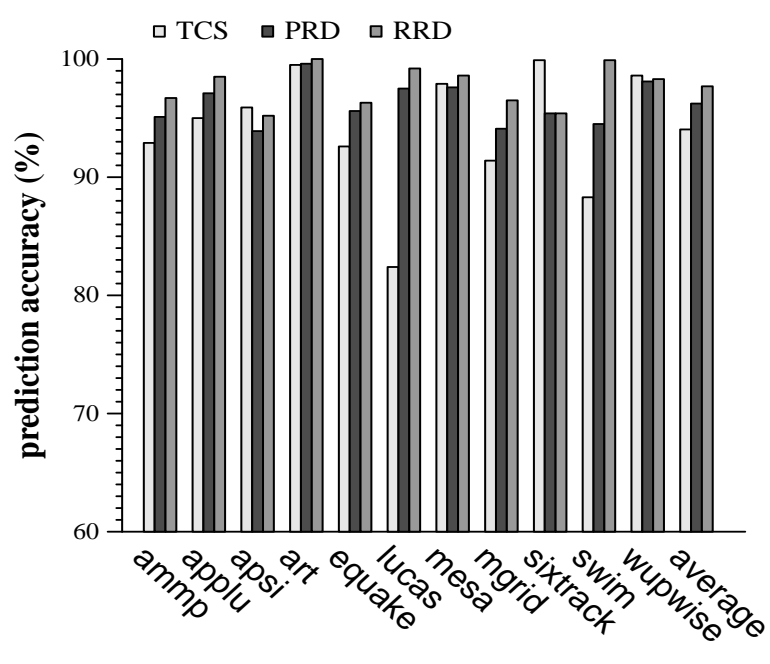

(a) L1 cache

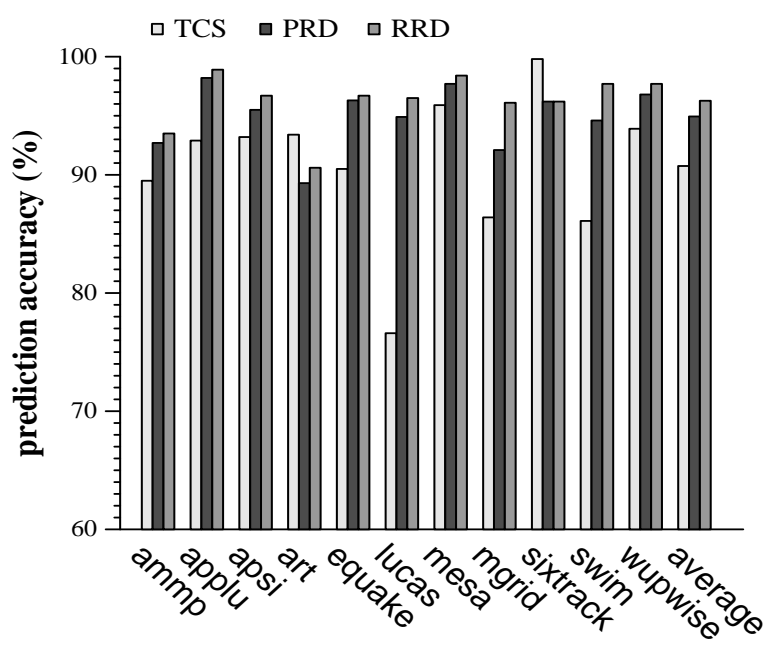

(c) 4-way set associative L2 cache

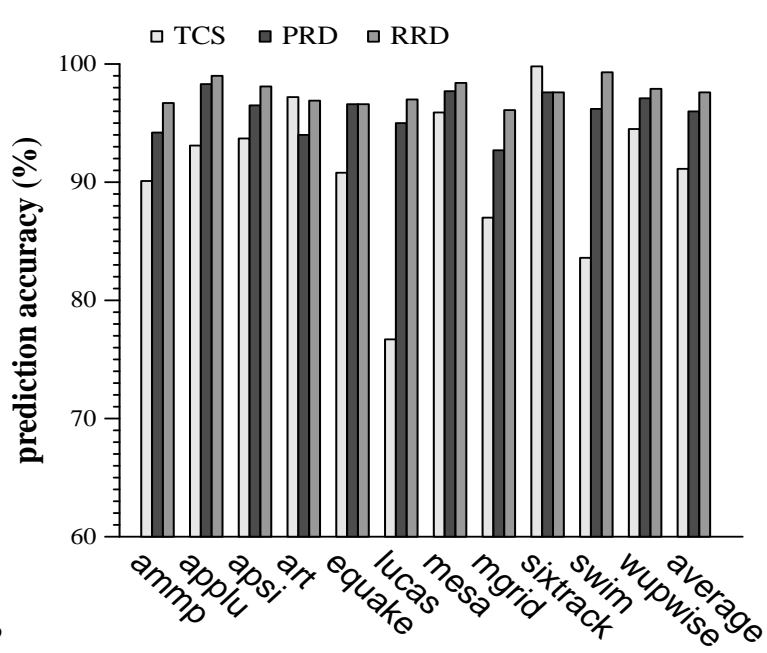

(b) Fully associative L2 cache

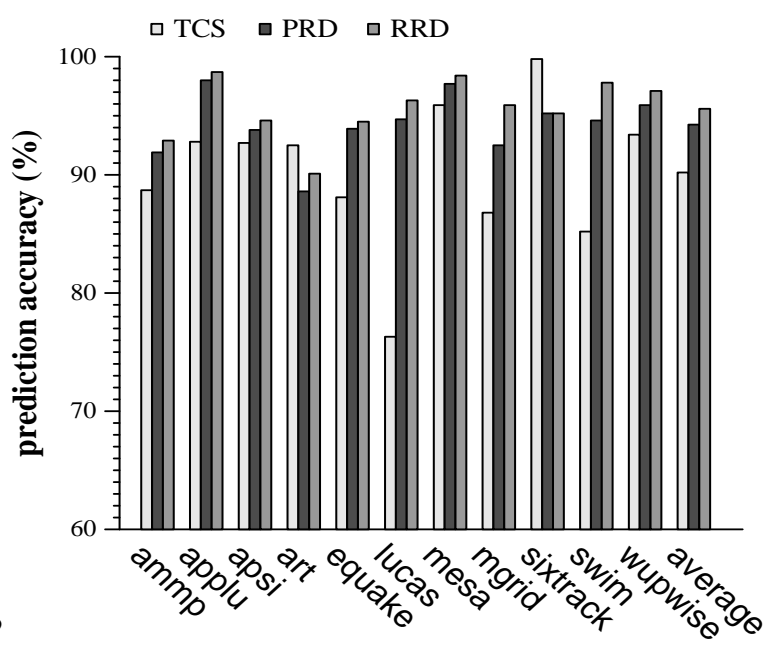

(d) 2-way set associative L2 cache

Figure 2: Static miss-rate prediction accuracy

\subsubsection{Miss-rate Prediction Accuracy}

Figures 1 and 2 report our miss-rate prediction accuracy using dynamic and static weighting, respectively, for cache configurations 1, 2 and 3 reported in Table 2. Table 7 summarizes the average miss rate prediction accuracy from those figures and the remaining cache configurations. These figures and table report the percentage of instructions whose miss rate is predicted within $5 \%$ of the actual miss rate. Examining Table 7 reveals that our prediction method (PRD) predicts the $\mathrm{L} 1$ miss rate of instructions with at least a $93.1 \%$ accuracy and the L2 miss rate with at least a $87.4 \%$ accuracy using the dynamic weighting. On average PRD more accurately predicts the miss rate than TCS, but is slightly less accurate than RRD.

Since TCS simply considers the test run's miss rate as the reference miss rate, TCS is only effective when an instruction has only constant patterns or the data sizes of the test and reference are very close (reuse distance change is minimal). PRD takes the data size change into account, and thus its prediction accuracy is not likely to be negatively influenced by a large change in input data size. For 189.lucas and 171.swim, the difference between the data sizes of test and reference is large, and both programs have a significant number of non-constant patterns. TCS makes a highly inaccurate prediction compared to $\mathrm{PRD}$, as shown in each part of Figures 1 and 2.

When the data size difference between test and reference runs is small (e.g. 200.sixtrack and 179.art), TCS can predict the miss rate quite accurately. TCS outperforms PRD for 200.sixtrack on the L1 miss rate slightly, and both setassociative L2 miss rates, but not the fully associative L2 miss rate. TCS even outperforms RRD in predicting miss rate on those same configurations. This is because the data size is not a factor and TCS can take into account conflict 


\begin{tabular}{|c|c|c|c|c|c|c|c|c|c|}
\hline \multirow{2}{*}{ Cache } & \multicolumn{3}{|c|}{$\mathbf{2 \%}$} & \multicolumn{3}{c|}{$\mathbf{5 \%}$} & \multicolumn{3}{c|}{$\mathbf{8 \%}$} \\
\cline { 2 - 11 } & TCS & PRD & RRD & TCS & PRD & RRD & TCS & PRD & RRD \\
\hline 2-way, 32K L1 & 90.4 & 95.0 & 97.2 & 90.6 & 95.6 & 97.3 & 90.7 & 96.0 & 97.3 \\
\hline 4-way, 1M L2 & 82.1 & 88.0 & 89.4 & 82.6 & 88.4 & 89.7 & 82.9 & 89.0 & 90.2 \\
\hline
\end{tabular}

Table 8: Prediction accuracy measures

\begin{tabular}{|l|r|}
\hline Benchmark & \% Intervals Merged \\
\hline 188.ammp & 14.9 \\
\hline 173.applu & 14.2 \\
\hline 301.apsi & 8.0 \\
\hline 179.art & 8.2 \\
\hline 183.equake & 7.0 \\
\hline 189.lucas & 7.2 \\
\hline 177.mesa & 7.6 \\
\hline 172.mgrid & 19.2 \\
\hline 200.sixtrack & 10.3 \\
\hline 171.swim & 9.7 \\
\hline 168.wupwise & 7.6 \\
\hline average & $\mathbf{1 0 . 4}$ \\
\hline
\end{tabular}

Table 6: Percentage of merged intervals

misses through simulation, whereas reuse distance cannot. 179.art exhibits some of the same patterns, but since the data size does change, PRD and RRD outperform TCS in some instances.

TCS also does well in L1 miss rate prediction for 301.apsi and 168.wupwise. For 168.wupwise, we believe this is due to the large percentage of constant reuse distance patterns, making data-set size change less pronounced. Note that for 168.wupwise PRD outperforms TCS on the L2 miss rates indicating that the constant patterns are more likely handled in the L1 cache and the changes in data size have more of an effect on the larger reuse distances. For 301.apsi, a large percentage of L1 miss are conflict misses. For small constant reuse patterns, TCS can predict those misses better than both PRD and RRD.

Figures 1(b) through 1(d) and Figures 2(b) through 2(d) illustrate our prediction accuracies for L2 misses for $1 \mathrm{M} \mathrm{L} 2$ caches with different associativities. These results show that PRD is effective in predicting L2 misses for a range of associativities. For fully-associative L2 cache, as shown in Figure 1(b) and 2(b), PRD predicts the miss rate correctly for $92.5 \%$ of the dynamically weighted instructions, and $96.2 \%$ of the statically weighted instructions correctly, while TCS predicts the miss rate with accuracies of only $84.0 \%$ and $91.2 \%$, respectively. Since there are no conflict misses for a fully-associative L2, PRD shows a significant improvement in the prediction over TCS for all programs except 179.art. In this instance, RRD outperforms TCS, while PRD does not. Since PRD is a heuristic, it does not always perform as expected. However, our results show that in most instances, PRD is quite effective.

Table 7 shows that across all cache configurations, on average PRD performs almost as well as RRD and better than TCS. These results indicate that PRD is not highly sensitive to cache size. Associativity does have an effect on PRD's prediction accuracy. Because lower associativity introduces more conflict misses, the accuracy will decrease. However, even for a 2-way set-associative L2 cache, on average, our model's prediction can still achieve $87.4 \%$ accuracy for dynamic weighting, and $94.5 \%$ for static weighting, leading us to believe that PRD shows much promise in future applications to cache optimization.

To examine the effects of our accuracy measure, we examine the miss-rate prediction accuracy if we considered a predicted miss rate to be accurate if that rate was within $2 \%, 5 \%$ and $8 \%$ of the acutal miss rate. Table 8 reports the dynamically weighted average miss rate prediction accuracy for the cache configuration 2 . As can be seen from Table 8 , the accuracy changes only slightly as we modify the prediction accuracy measure. Note that there are no individual programs where the changes are more dramatic than reported in the averages.

\subsubsection{Critical Instructions}

For static or dynamic optimizations, we are interested in the critical instructions which generate the most L2 misses. In this section, we show that we can predict most of the critical instructions. We also observe that the locality intervals of the critical instructions tend to be more diverse than noncritical instructions and tend to exhibit non-constant reuse patterns more frequently.

We perform cache simulation on the reference input using cache configuration 2 to identify the critical instructions which generate the most L2 misses: those static instructions whose cumulative L2 misses account for 95\% of the total L2 misses in the program. To predict critical instructions, we use the execution frequency in one training run to estimate the relative contribution of the number of misses for each instruction given the total miss rate. We then compare the predicted critical instructions with the real ones and show the prediction accuracy weighted by the absolute number of misses in Table 9. We also list the number of critical instructions for each benchmark in the column labeled "\# Critical" and the total number of static memory operations for each benchmark in the column labeled "Total".

The prediction accuracy for critical instructions is $86 \%$ on average. 189.lucas shows a very low accuracy because of low prediction coverage. The unpredictable instructions in 189.lucas contribute a significant number of misses. Notice that the number of critical instructions in most programs is very small. These results show that reuse distance can be used to allow compilers to target the most important instructions for optimization effectively.

Critical instructions tend to have more diverse locality patterns than non-critical instructions. Table 10 reports the distribution of the number of locality intervals for critical instructions using dynamic weighting. We find that the distribution is more diverse than that shown in Table 4. Although less than $3 \%$ of the instructions on average have more than 2 intervals, the average goes up to over $23 \%$ when considering only critcial instructions. However, only a small number of critical instructions have more than three intervals and no instructions exhibit over six intervals.

Critical instructions also tend to exhibit a higher percentage of non-constant patterns than non-critical instructions. 


\begin{tabular}{|l|r|r|r|}
\hline Benchmark & Accuracy & \# Critical & Total \\
\hline 188.ammp & 85.2 & 16 & 5180 \\
\hline 173.applu & 93.0 & 224 & 11352 \\
\hline 301.apsi & 84.7 & 194 & 11567 \\
\hline 179.art & 99.5 & 14 & 1820 \\
\hline 183.equake & 87.7 & 76 & 3018 \\
\hline 189.lucas & 57.3 & 201 & 2915 \\
\hline 177.mesa & 100.0 & 3 & 5736 \\
\hline 172.mgrid & 76.0 & 84 & 2185 \\
\hline 200.sixtrack & 93.2 & 85 & 19688 \\
\hline 171.swim & 99.4 & 263 & 2028 \\
\hline 168.wupwise & 72.7 & 15 & 1667 \\
\hline average & 86.2 & 107 & 6105 \\
\hline
\end{tabular}

Table 9: Prediction accuracy for critical instructions

\begin{tabular}{|l|l|l|l|l|l|l|}
\hline Benchmark & \multicolumn{7}{|c|}{ Interval Distribution (\%) } \\
\hline & 1 & 2 & 3 & 4 & 5 & 6 \\
\hline 188.ammp & 96.3 & 0.0 & 3.7 & 0.0 & 0.0 & 0.0 \\
\hline 173.applu & 6.0 & 77.9 & 16.1 & 0.0 & 0.0 & 0.0 \\
\hline 301.apsi & 7.0 & 58.5 & 30.7 & 3.7 & 0.0 & 0.0 \\
\hline 179.art & 100.0 & 0.0 & 0.0 & 0.0 & 0.0 & 0.0 \\
\hline 183.equake & 18.8 & 80.0 & 1.2 & 0.0 & 0.0 & 0.0 \\
\hline 189.lucas & 7.9 & 92.1 & 0.0 & 0.0 & 0.0 & 0.0 \\
\hline 177.mesa & 0.0 & 100.0 & 0.0 & 0.0 & 0.0 & 0.0 \\
\hline 172.mgrid & 0.0 & 67.3 & 28.1 & 3.0 & 0.6 & 1.0 \\
\hline 200.sixtrack & 0.3 & 18.8 & 79.4 & 1.4 & 0.0 & 0.0 \\
\hline 171.swim & 0.0 & 98.8 & 1.2 & 0.0 & 0.0 & 0.0 \\
\hline 168.wupwise & 0.4 & 10.1 & 68.4 & 5.4 & 15.7 & 0.0 \\
\hline average & 21.5 & 54.9 & 20.8 & 1.2 & 1.5 & 0.1 \\
\hline
\end{tabular}

Table 10: Distribution of the number of locality intervals for critical instructions

Table 11 reports the percentage of critical instructions that have all constant patterns. As can be seen, critical instructions are more sensitive to data size. This fact makes it important to predict accurately reuse distance in order to apply optimization to the most important memory operations. Note that we report no data for 200.sixtrack because all of its data sizes are identical.

\begin{tabular}{|l|r|}
\hline Benchmark & \% Constant \\
\hline 188.ammp & 25.0 \\
\hline 173.applu & 13.8 \\
\hline 301.apsi & 7.7 \\
\hline 179.art & 50.0 \\
\hline 183.equake & 11.8 \\
\hline 189.lucas & 1.5 \\
\hline 177.mesa & 0.0 \\
\hline 172.mgrid & 35.7 \\
\hline 200.sixtrack & - \\
\hline 171.swim & 0.0 \\
\hline 168.wupwise & 33.3 \\
\hline average & $\mathbf{1 7 . 9}$ \\
\hline
\end{tabular}

Table 11: Percentage of critical instructions having all constant patterns

\section{CONCLUSIONS AND FUTURE WORK}

In this paper, we have demonstrated that reuse distance is predictable on a per instruction basis for floating-point programs. On average, over $90 \%$ of all memory operations executed in a program are predictable with an almost $97 \%$ accuracy. In addition, the predictable reuse distances translate to predictable miss rates for the instructions. For a
32KB 2-way set associative cache, our miss-rate prediction accuracy is almost $96 \%$, and for a $1 \mathrm{MB}$ fully associative L2 cache, our miss-rate prediction accuracy is over $92 \%$. Our analysis also identifies the critical instructions in a program that contribute to $95 \%$ of the program's L2 misses. On average, our method predicts the critical instructions with an $86 \%$ accuracy.

The next step in our research is to apply reuse-distance and miss-rate prediction to cache optimization. We are currently developing a hardware-based prefetching mechanism that will utilize reuse distance for those instructions likely to cause a significant number of cache misses. In addition, we are beginning work on phase detection of reuse distance. If an instruction has multiple reuse-distance intervals, we would like to be able to determine if those reuse distance intervals occur in phases. Detecting phases will allow us to further target cache optimization and possibly use sourcelevel transformations to isolate the regions in the code where misses are likely to occur. Additionally, we plan to investigate the use of reuse distance in optimizing pointer-based applications.

In order for significant gains to be made in improving program performance, compilers must improve the performance of the memory hierarchy. Our work is a step in opening up new avenues of research through the use of feedbackdirected and dynamic optimization in improving program locality through the use of reuse distance.

\section{ACKNOWLEDGMENTS}

This work was partially supported by NSF grant CCR0312892. The authors would like to thank the anonymous referees for their suggestions to improve this paper.

\section{REFERENCES}

[1] S. Chatterjee, E. Parker, P. J. Hanlon, and A. R. Lebeck. Exact analysis of the cache behaviour of nested loops. In Proceedings of the SIGPLAN 2001 Conference on Programming Language Design and Implementation, pages 286-297, Snowbird, Utah, June 2001.

[2] C. Ding and Y. Zhong. Predicting whole-program locality through reuse distance analysis. In Proceedings of the 2003 ACM SIGPLAN Conference on Programming Language Design and Implementation, pages 245-257, San Diego, California, June 2003.

[3] S. Ghosh, M. Martonosi, and S. Malik. Precise miss analysis for program transformations with caches of arbitrary associativity. In Proceedings of the Eighth International Conference on Architectural Support for Programming Languages and Operating Systems, pages 228-239, San Jose, CA, Oct. 1998.

[4] G. Goff, K. Kennedy, and C. Tseng. Practical dependence testing. In Proceedings of the SIGPLAN '91 Conference on Programming Language Design and Implementation, pages 15-29, Toronto, Canada, June 1991.

[5] K.Belys and E. D'Hollander. Reuse distance-based cache hint selection. In Proccedings of the 8th International Euro-Par Conference, August 2002.

[6] R. L. Mattson, J. Gecsei, D. Slutz, and I. L. Traiger. Evaluation techniques for storage hierarchies. IBM Systems Journal, 9(2):78-117, 1970. 
[7] K. S. McKinley. A compiler optimization algorithm for shared-memory multiprocessors. IEEE Transactions on Parallel and Distributed Systems, 9(8):769-787, Aug. 1998.

[8] K. S. McKinley, S. Carr, and C. Tseng. Improving data locality with loop transformations. $A C M$ Transactions on Programming Languages and Systems, 18(4):424-453, July 1996.

[9] K. S. McKinley and O. Temam. Quantifying loop nest locality using SPEC'95 and the Perfect benchmarks. ACM Transactions on Computer Systems, 17(4):288-336, Nov. 1999.

[10] W. Pugh. A practical algorithm for exact array dependence analysis. Communications of the ACM, 35(8):102-114, Aug. 1992.

[11] A. Srivastava and E. A. Eustace. Atom: A system for building customized program analysis tools. In Proceeding of ACM SIGPLAN Conference on Programming Language Design and Inplementation, June 1994.

[12] R. A. Sugumar and S. G. Abraham. Efficient simulation of caches under optimal replacement with applications to miss characterization. In Proceedings of the ACM SIGMETRICS Conference on Measurement \& Modeling Computer Systems, pages 24-35, Santa Clara, CA, May 1993.

[13] M. E. Wolf and M. Lam. A data locality optimizing algorithm. In Proceedings of the SIGPLAN'91 Conference on Programming Language Design and Implementation, pages 30-44, Toronto, Canada, June 1991.

[14] Y. Zhong, S. Dropsho, and C. Ding. Miss rate prediction across all program inputs. In Proceedings of the $12^{\text {th }}$ International Conference on Parallel Architectures and Compilation Techniques, pages 91-101, New Orleans, LA, September 2003. 